\title{
Les chemins de fer et l'heure légale
}

\section{Lucien Baillaud}

\section{CpenEdition}

\section{Journals}

Édition électronique

URL : https://journals.openedition.org/rhcf/416

DOI : $10.4000 /$ rhcf. 416

Éditeur

Rails \& histoire

Édition imprimée

Date de publication : 1 novembre 2006

ISSN : 0996-9403

Référence électronique

Lucien Baillaud, «Les chemins de fer et l'heure légale », Revue d'histoire des chemins de fer [En ligne], 35 | 2006, mis en ligne le 05 avril 2011, consulté le 22 avril 2022. URL : http://journals.openedition.org/ rhcf/416 ; DOI : https://doi.org/10.4000/rhcf.416 


\section{Lucien BAILLAUD}

Ancien président du Groupe d'étude des rythmes biologiques, Société francophone de chronobiologie Institut pour la gestion et la valorisation des herbiers universitaires de Clermont-Ferrand

\section{Les chemins de fer et l'heure légale}

La notion d'heure repose sur plusieurs bases d'ordres très différents : le point de vue de l'astronomie, avec la succession des jours et des nuits; les modalités de l'adaptation de notre organisme et de la société aux contraintes du cycle jour-nuit, cycle nycthéméral ; un aspect de pure convention, le découpage du temps en compartiments et leur désignation : la numérotation des heures; la technique de l'horlogerie, avec ses possibilités et ses progrès ; enfin les règlements de la société, la loi, hors laquelle il n'y a pas d'heure « légale ».

Dans un lieu donné, « midi » désigne le milieu de la journée, à la mi-temps du lever et du coucher du soleil. On a longtemps, en toutes saisons, divisé le jour en 12 heures et la nuit aussi, heures inégales sauf aux équinoxes, mais l'invention des horloges à poids a permis de donner la même durée aux heures du jour et de la nuit (heures équinoxiales). D’un « midi » au suivant il s'écoule une durée un peu variable, d'environ 24 de nos « heures », le « jour solaire vrai »; l'observation du ciel donne $l^{\prime}$ « heure locale vraie ». Pour ne pas demander aux horlogers de construire des horloges à vitesse variable selon les époques de l'année, on a privilégié un « temps moyen » artificiel, établi d'après la moyenne des jours solaires ; la première décision officielle a été prise à Genève le $1^{\text {er }}$ janvier $1780^{1}$. Il peut y avoir une différence d'une quinzaine de minutes, d'avance ou de retard, entre l'heure du temps moyen et l'heure vraie. C'est l'heure moyenne qui scande notre vie, si nous nous référons au temps des horloges plutôt qu'à la nature.

\section{Les inconvénients des heures locales}

Cela aboutissait à une notion d'heure locale rationnelle, commode, mais il y a sur notre planète autant d'heures locales que de longitudes différentes : pour aussi innombrables qu'elles soient, elles

1- Walter ZURBUCHEN, «Quelle heure est-il ?», Revue du Vieux Genève, 1976, p. 20-21. 
étaient adaptées à une population sédentaire. Elles sont devenues gênantes avec le développement des communications rapides vers le milieu du XIX ${ }^{\mathrm{e}}$ siècle.

Une documentation considérable a été publiée sur le sujet par des astronomes et par des spécialistes des transports. Parmi les travaux les plus substantiels ${ }^{2}$, citons en 1885 celui de Sanford Fleming, chef des voies de communication du Canada, et le livre de Derek Howse (1980), directeur de la navigation et de l'astronomie du National Maritime Museum de Greenwich.

Il fallait prendre conscience des inconvénients des heures locales, inventer un temps à valeur géographiquement plus étendue, poursuivre au plan national puis international l'uniformisation de l'heure, imaginer une «heure standard», trouver les moyens pratiques d'obtenir cette uniformisation, convaincre de son opportunité les personnalités-clés et passer à la réalisation.

Les médias de cette époque n'avaient pas une diffusion rapide, de sorte que les idées lancées ici ou là pouvaient n’avoir aucun écho immédiat ailleurs. Cependant, une fois les problèmes résolus tout parait aller de soi; pour l'usager, les horaires des chemins de fer sont des données évidentes de la vie courante. On regrette d'autant plus, ici, la nécessité d'être bref pour rendre compte de la diversité des questions soulevées, techniques et humaines.

Depuis le XVIII ${ }^{e}$ siècle et le travail de mécanique horlogère de Pierre Le Roy, Ferdinand Berthoud et John Harrison, les marins, pour faire le point en mer, emportaient des chronomètres garde-temps, qui leur fournissaient en permanence l'heure d'une localité du globe aux coordonnées astronomiques bien déterminées. Ils voyageaient avec, par exemple, l'heure de Paris, de Cadix ou de Greenwich dans leur matériel de navigation. On a aussi proposé de transporter «l'heure » au cours de trajets terrestres, mais l'objectif était différent; dès 1840, en effet, le capitaine Basil Hall (1788-1884) eut l'idée de faire marquer par tous les

\footnotetext{
2- Sanford FLEMING et al., Universal or cosmic time, together with other papers, Toronto, Council of the Canadian Institute, 1885, vi +84 p. ; Guillaume BIGOURDAN, « Le jour et ses divisions. Les fuseaux horaires et l'Association internationale de l'heure ", Annuaire du bureau des longitudes 1914, p. B.1-B.107 ; H.M. SMITH, "Greenwich time and the prime meridian ", Vistas in Astronomy, 20, 1976, p. 219-229 ; Derek HOWSE, Greenwich time and the discovery of the longitude, Oxford University Press, 1980, 254 p. ; Gérard JASNIEWICZ, "La détermination et la conservation de l'heure, histoire d'une fonction sociale ", Publications de l'observatoire astronomique de Strasbourg, série astronomie et sciences humaines, 1, 1988, p. 59-64.
} 
bureaux de poste britanniques la même heure, qui serait donnée par des chronomètres apportés de Londres par la malle-poste. En 1842, un rapport était publié, sur la proposition du météorologiste Follett Osler, demandant l'instauration d'une heure commune pour toute la Grande-Bretagne. Ce fut presque réalisé en une décennie.

L'époque des diligences céda la place à l'ère des chemins de fer. À moins de circuler selon des arcs de méridiens, les trains passaient par des stations dont les heures locales étaient différentes les unes des autres. Or ils fonctionnaient avec des horaires réguliers : les responsables de la marche de chaque train devaient savoir à quelle heure il fallait arriver à tel ou tel endroit ; la situation risquait d'être intenable : on régla chaque ligne en fonction de l'heure d'une seule localité, celle d'un terminus ; en France, ce fut l'heure de Paris. Cela n'annulait pas la dualité avec l'heure, locale, utilisée par le voyageur montant dans le train.

Comment s'organiser par rapport à cette dualité ? Le cas de l'Allemagne présente un intérêt particulier, du fait du nombre des États qui composaient cet empire. Les heures locales des diverses métropoles (Berlin, Dresde, Cologne, etc.) servaient d'heures régulatrices pour les diverses lignes de chemins de fer. On unifia les choses pour toute l'Allemagne du Nord et l'Elsass-Lothringen; cependant le règlement du 4 janvier 1875 exigeait que chaque station ait une horloge réglée sur l'heure locale et que les guides horaires destinés au public donnent l'heure locale de chacune des localités desservies ; à en croire les horaires annoncés, il fallait près d'une heure de plus pour aller de Cologne à Berlin que de Berlin à Cologne : les longitudes en étaient la cause. Les conducteurs de trains n'avaient pas à connaitre le temps local, ils se guidaient d'après leurs propres chronomètres, en se conformant aux horaires de service établis pour eux, différents des horaires destinés au public.

Dans presque toute l'Europe, les compagnies ont pris pour heure normale celle de la capitale. Toutefois, la Suède, à la capitale trop excentrique, se régla sur le méridien de $15^{\circ}$ à l'est de Greenwich, une heure ronde de décalage, comme par prémonition des fuseaux horaires ; la Bavière, à l'aire disjointe, prit l'heure de Ludwigshafen et celle de Munich ; l'Italie se réglait sur Turin, Florence, etc. ; l'Autriche-Hongrie et la Russie avaient de multiples références horaires. Ces heures centralisées ne concernaient d'abord que les chemins de fer, mais le rôle croissant de ces derniers dans la vie de la société provoqua partout les mêmes nécessités qu'en Grande-Bretagne ; les divers États européens unifièrent peu à peu les heures de leur vie civile en les calquant sur celle de la gare. Il ne s'agissait pas toujours d'heure «légale »; ce n'était pas toujours la 
loi qui définissait l'heure, mais bien les astronomes, en scrutant le ciel, même si une somme d'initiatives locales se traduisait par quelques coups de pouce.

\section{Vers I'unification des heures: les heures nationales}

Pour unifier l'heure, il faut pouvoir la transmettre, si possible sans devoir transporter les horloges elles-mêmes. Suzanne Débarbat rappelle que l'amiral Ernest Mouchez, à l'Observatoire de Paris, pouvait envoyer l'heure par le télégraphe (devenu électrique) et aussi par la synchronisation directe $^{3}$. Cela permit l'unification de l'heure dans Paris (1881).

Ce sont bien les chemins de fer de France qui nous intéressent ici spécialement, mais les lignes de tous les pays étaient tributaires les unes des autres.

En opposition au système, compliqué, qui s'est développé en Allemagne, les compagnies britanniques avaient commencé, dès novembre 1840, de mettre les horloges des gares à l'heure de Londres et de chiffrer les horaires des trains par rapport au temps de Londres. En 1847 un organisme de coordination des chemins de fer recommandait de prendre en compte l'heure de l'Observatoire royal voisin, Greenwich ; la différence était de 23 secondes.

Dans toutes les localités où les trains s'arrêtaient, régnait, bien sûr, une heure locale, censée être celle de la vie ordinaire des gens ; on y ajoutait l'heure de la gare, celle des trains, qui était l'heure de Greenwich. Mais les habitants prirent l'habitude de se référer à l'heure de la gare pour la vie civile. Howse signale que quelques villes occidentales de la GrandeBretagne, éloignées du méridien de Greenwich, conservèrent leurs heures locales plus longtemps que les autres; mais il affirme qu'en $185598 \%$ des horloges publiques britanniques étaient à l'heure de Greenwich.

En France, cette évolution, initialement provoquée par les besoins des chemins de fer, conduisait vers l'uniformité de la notation du temps, d'une longitude à l'autre, pour l'ensemble de la société. L'Observatoire de Paris diffusa l'heure parisienne à Rouen (1880), au Havre un an plus tard, puis à La Rochelle, Nancy, etc. ; à Besançon un observatoire déterminait l'heure locale, mais depuis le $1^{\text {er }}$ janvier 1890 la municipalité lui demanda de donner l'heure du méridien de Paris, pour régler les horloges publiques de la ville sur «l'heure nationale»: les Bisontins appelaient ainsi l'heure des chemins de fer.

3- Suzanne DÉBARBAT, «1891. L'heure de Paris devient l'heure nationale », Célébrations nationales, Paris, Direction des Archives de France, 1991, p. 97-98. 


\section{L'heure de Rouen}

Jusqu'en 1891 la situation en France ressemblait à ce qui se passait initialement en Grande-Bretagne ; il y avait l'heure locale et l'heure de la gare, qui était celle du temps moyen de la capitale. On voyait ces deux heures sur les cadrans extérieurs des gares et dans les cours et salles de départ. Mais on connait la plaisanterie «qui trop embrasse manque le train » : les voyageurs habitués aux diligences avaient peut-être la hantise des horaires minutés des trains ; en fait, ils avaient tendance à arriver en avance, comme aujourd'hui dans les aéroports ; quant aux responsables des chemins de fer, afin d'éviter d'éventuelles réclamations des voyageurs, ils introduisirent un perfectionnement supplémentaire, pour aider les voyageurs à ne pas "manquer le train »: on mit tous les trains en retard par rapport aux horaires annoncés ${ }^{4}$; sur les trottoirs de départ, on lisait une troisième " heure », en retard par rapport à l'heure officielle « de la gare » et sur laquelle les trains se réglaient effectivement. Au début ce décalage était de 3 minutes dans le réseau du Nord et de 5 dans les autres; en 1887 on unifia le retard à 5 minutes pour tous les réseaux ; en Belgique, il était de 5, 7 ou 10 minutes. Louis De Busschère (1891) cite Wilhem de Nordling, pour qui ce décalage était destiné «à donner des jambes aux voyageurs attardés ».

Ainsi donc, les chemins de fer, en France, étaient réglés non pas sur les heures locales de la vie de tout le monde, ni sur l'heure « de la gare ", heure de Paris généralisée, mais sur le méridien situé à 5 minutes de retard de temps, soit $1^{\circ} 15^{\prime}$ de longitude ouest de Paris, le méridien de Rouen. Pour une localité donnée, le départ d'un même train se faisait à trois heures nominales différentes. Joseph Rocca soulignait que l'heure de Rouen réglait le plus important des services publics. Cette particularité française fut supprimée le 11 mars 1911.

Entre ces diverses mesures et la promulgation de l'heure légale, des avancées essentielles se sont déroulées en Amérique, grâce aux "Américains du Nord, avec leur admirable sens pratique de Business" $m e n »^{5}$.

4- Louis DE BUSSCHÈRE, « Note sur la situation actuelle de l'unification des heures », Bulletin de la commission internationale du congrès des chemins de fer, avril-juin 1891, p. 138-166.

5- Charles LALLEMAND, «L'unification des heures et le système des fuseaux horaires », Revue scientifique, avril 1897, p. 419-425. 


\section{Le système américain}

En Amérique du Nord, jusqu'en 1884 les horaires des trains étaient établis, comme initialement en Europe, d'après l'heure locale de la ville la plus importante de la ligne ou d'une ville terminus. Dans une même salle de gare, plusieurs horloges indiquaient des heures différentes selon les lignes concernées, en plus de l'heure locale que le voyageur avait peut-être sur sa montre. Les quelque 75 heures locales utilisées dans l'ensemble des États-Unis rendaient compliquée la consultation des guides des chemin de fer. Le chef de gare doit chiffrer différemment le départ d'un même train s'il s'adresse au personnel du train ou au personnel de la gare ou au public ${ }^{6}$.

Qui a inventé le système des fuseaux horaires et de l'heure standard ? Pour Nordling (1890), «Il serait difficile de dire qui en a eu la première idée, tant elle est naturelle, mais il est certain que ce sont les Américains qui, les premiers, l'ont appliqué en grand [...] $»^{7}$. Pour Allen (1884), cité par Louis De Busschère (1890) : " almost every city in the country has recently discovered that within its borders dwelt the Father of standard time. ${ }^{8}$." En fait, des propositions concernant l'heure ont été émises par une dizaine d'auteurs, si ce n'est plus. Selon Nordling (1890) « l'auteur principal du système » aurait été Sandford Fleming, « alors ingénieur en chef du chemin de fer Transcontinental du Canada ». Mais depuis longtemps personne ne conteste plus que la priorité revienne à Charles Ferdinand Dowd qui, lui, n'était pas un cheminot, ce qui pouvait nuire à son image auprès des professionnels et à son aptitude à les convaincre.

La ville de Saratoga Springs, dans l'État de New York, se targue d'être celle où C.F. Dowd a inventé l'heure standard et les fuseaux horaires ${ }^{9}$. Dowd était né à Madison (Connecticut) le 25 avril 1825 ; il avait obtenu en 1853 un PhD de théologie à l'Université Yale, à New Haven, ce qui lui valait le titre de Doctor ou de Reverend. C'était un « Presbyterium clergyman ».

\footnotetext{
6- Wilhem de NORDLING, "L'unification des heures », Revue générale des chemins de fer, avril 1888, p. 193-212.

7- Wilhem de NORDLING, «L'unification des heures », Bulletin de la société de géographie, série 7, 10 (1 $1^{\text {er }}$ trim. 1890), p. 111-137.

8- Louis DE BUSSCHÈRE, art. cité.

9- Charles Ferdinand DOWD, System of national time for rail-roads; a specimen table and a time gazetteer for all stations in the United States and Canada, 1870, Albany, Weed, Parsons and Company, 107 p. ; C.N. DOWD, Charles F. Dowd, AM, Ph.D. and standard time, New York, Knickerbocker Press, 1930.
} 
De 1865 à 1898, il dirigeait une école pour jeunes filles, le Temple Grove Ladies Seminary, remplacé actuellement par le Skidmore College. Après une vie largement consacrée à rendre service aux chemins de fer, il mourut le 12 novembre 1904, ingrate fatalité ferroviaire, sous les roues d'une locomotive à un passage à niveau de Saratoga Springs ${ }^{10}$.

Il n'y avait aucun lien entre la profession de C.F. Dowd et son intérêt pour les problèmes de la désignation de l'heure, mais c'était un homme d'ordre. Il savait que les chemins de fer anglais étaient réglés d'après une heure unique, celle de la capitale ou plutôt celle de l'Observatoire de Greenwich. Il pensait que cela n'était pas réalisable aux États-Unis à cause des distances trop grandes (à peu près quatre heures de différence entre les heures locales des deux côtes océaniques du pays). Il eut l'idée d'une division selon des méridiens, en quatre sections qui différeraient l'une de l'autre de $15^{\circ}$ de longitude et seraient décalées d'une heure ronde ; les horloges réglant les horaires des trains de tous les États-Unis marqueraient toutes la même minute.

Il présenta son idée en octobre 1869 à une assemblée de responsables des «Trunk Lines » à New York. Le comité de cette assemblée approuva le principe, mais demanda comment cela pourrait se réaliser en détail. À l'automne de 1870, Dowd publia une brochure donnant la correction des heures locales de plus de 8000 localités et une carte représentant les zones horaires qu'il préconisait. L'ensemble était rapporté, comme cela avait été le cas en Angleterre, à une longitude nationale, en l'occurrence celle de Washington. Les Trunk Lines ne le prirent pas en considération.

Dowd multiplia les interventions auprès des autres organisations. Le comité d'Atlanta, en Géorgie, proposa de remplacer la référence de Washington par New York; cela entrainait un décalage de $3^{\circ}$ de longitude des méridiens repères, soit 12 minutes de temps dans les décalages des heures nominales, et c'était plus commode pour certaines sections. Mais pour des raisons d'universalisme Dowd ne souhaitait pas abandonner un méridien national au profit d'un méridien local ; par ailleurs New York est à $74^{\circ} 1^{\prime}$ de longitude ouest par rapport à Greenwich ; Dowd aimait mieux se référer au chiffre rond, au $75^{\mathrm{e}}$ méridien à l'ouest de Greenwich, soit moins de 4 minutes de temps par rapport à New York; ce choix heurtait les ressentiments des Américains à l'égard de leur ancienne métropole, mais il avait l'avantage de tout rapporter au système de méridiens adopté par la marine américaine. Dowd continua ses démarches sur ces bases.

10- Wall Street daily News : «November 16, 1904, Charles Ferdinand Dowd. » 
Les responsables des sociétés ferroviaires tergiversaient, notant que beaucoup de voyageurs ne faisaient que de courts trajets. Ils penchaient pour une adoption ligne par ligne, chaque compagnie agissant librement. Dowd chercha à convaincre les entreprises une par une. Le problème commençait d'ailleurs à être dans l'air du temps. Ainsi, en 1879, Sanford Fleming adressait à l'Académie de Toronto un mémoire proposant d'une part comme méridien initial celui du détroit de Bering et d'autre part la division du globe en 24 fuseaux de $15^{\circ}$ chacun en longitude. En mai 1879, intervint la Metrological Society : il y eut un comité de l'heure standard, présidé par Cleveland Abbé, qui étudia le sujet. D'un autre côté, l'American Railway Association confia la question à son secrétaire, William F. Allen, l'éditeur du Travelers Official Railway Guide, qui présenta son rapport à Saint Louis en avril 1883 ; il proposait le système de Dowd.

La General Time Convention groupait les délégués de 35 réseaux du Canada et du Nord des États-Unis. Elle se réunissait deux fois par an. En avril 1883, à Saint Louis, elle étudia longuement la question. Le 11 octobre 1883, à Chicago, elle décida l'adoption des fuseaux horaires, réglés sur Greenwich, qui entrèrent en vigueur le 18 novembre. Le 17 octobre, à New York, la Southern Time Convention (concernant 34 compagnies des États du Sud) prit la même décision, qui fut appliquée le 20 novembre.

Il s'agissait de la dénomination des heures, à l'usage des chemins de fer seulement. Cela ne changeait rien ni à la réalité des horaires des trains, ni à la vie courante des personnes. Cependant les Nord-Américains adoptèrent très vite l'heure de leur fuseau, l'heure des chemins de fer, à la place des heures locales, pour l'ensemble de la vie civile. Quatre mois après les décisions des compagnies, le 9 avril 1884 William F. Allen affirmait que $90 \%$ des Américains avaient abandonné leurs heures locales ${ }^{11}$. Depuis le 14 mars 1884, de par la loi, Washington était à l'heure du méridien $75^{\circ}$ ouest de Greenwich.

\section{La conférence de Washington}

L'Europe ne se préoccupait pas des débats ferroviaires américains, mais les astronomes et les géodésiens souhaitaient unifier leurs notations horaires et celles des longitudes. L'association géodésique tint sa $7^{\mathrm{e}}$ conférence à Rome le 15 octobre 1883 ; elle optait pour Greenwich comme méridien origine et pour une heure qui serait celle de Greenwich comptée de 0 à 24 avec 0 à minuit

11- Louis DE BUSSCHÈRE, art. cité. 
moyen. Ces recommandations, d'ordre scientifique, ne concernaient pas la vie sociale, mais la conférence souhaitait que l'heure de Greenwich soit utilisée par le service intérieur des chemins de fer et par les postes et télégraphes ; elle souhaitait un accord international.

Retour en Amérique. Le gouvernement des États-Unis provoqua la réunion à Washington d'un congrès diplomatique et scientifique, qui se tint du $1^{\text {er }}$ octobre au $1^{\text {er }}$ novembre 1884 à Washington, en vue d'une entente sur ces questions ${ }^{12}$. La France était représentée à l'International Meridian Conference par un ministre plénipotentiaire et par l'astronome Jules Janssen, qui souhaitait l'adoption non pas du méridien de Paris, rêve utopique, ni surtout de celui de Greenwich, abhorré, mais d'un méridien neutre, vers les Açores ou le détroit de Bering. Cela aurait conduit à réajuster les habitudes de tous les marins du monde et à rectifier toutes les cartes existantes.

Or, coïncidence étonnante, la « General Time Convention » des chemins de fer se réunissait au même moment à Philadelphie. Le 9 octobre 1884, elle votait une motion informant la conférence internationale des méridiens réunie à Washington que le système basé sur l'heure des méridiens de 75, 90, 105 et $120^{\circ}$ de longitude ouest de Greenwich était entièrement satisfaisant et qu'il serait inopportun d'adopter un autre méridien initial. Allen lui-même était parmi les délégués des États-Unis à la Conférence de Washington. C'est donc à Washington que les délégués européens (en tout cas les Français) apprirent l'invention géniale des fuseaux horaires, compromis idéal entre l’heure universelle et les heures locales : en Amérique, sa réalisation était un fait accompli, avec quatre fuseaux en usage.

Pour revenir au programme de la conférence, des votes à de très fortes majorités approuvèrent d'abord l'idée d'un méridien initial unique, puis que ce soit celui du centre de l'instrument méridien de l'Observatoire de Greenwich, que les longitudes soient comptées à partir de ce méridien, que le jour universel soit un jour solaire moyen commençant à minuit moyen du premier méridien (au moment du début du jour civil et du changement de date sur ce méridien), et que le jour soit compté de 0 à 24 heures. Enfin les délégués français, sûrs de la haute valeur de ce que la France avait apporté à l'humanité, souhaitaient la généralisation du système décimal aux angles et aux longitudes ; la conférence se contenta

12- Jules JANSSEN, " Notice sur le méridien et l'heure universels », Annuaire du bureau des longitudes 1886 , p. 835-881 ; Louis DE BUSSCHÈRE, "Note sur l'unification des heures au point de vue de l'exploitation des chemins de fer", Bulletin de la commission internationale du congrès des chemins de fer, mars 1890, p. 408-442, pl. III-IV. 
de demander qu'on reprenne les études dans ce sens. La conférence n'avait pas pouvoir de décision; elle n'a pas été suivie d'un accord international officiel. Par ailleurs la question des fuseaux horaires, évoquée au cours des séances, n'apparait pas dans les conclusions, mais elle relevait de l'évidence et les débats ne portaient plus que sur la désignation des 24 fuseaux (numéros, lettres ou termes géographiques).

\section{Vers la généralisation des fuseaux horaires}

Après la conférence de Washington, il paraissait logique d'adapter à l'Europe le «système américain » ${ }^{13}$. La Grande-Bretagne réglait déjà ses chemins de fer et sa vie courante sur l'heure de Greenwich; elle n’avait qu'à maintenir le statu quo. La Suède était, par avance, déjà réglée sur le fuseau de l'Europe centrale. Les autres États avaient chacun une heure nationale ; il s'agissait non seulement de l'abandonner, mais que ce soit au profit, si l'on peut dire, de l'heure d'un autre État, qui passait pour arrogant ( $($ Rule, Britannia the World »).

En 1888, Nordling signalait que le Bureau des longitudes préparait un projet de loi visant à remplacer dans toute la France le temps moyen local, en vigueur à l'époque, par l'heure de Paris ; cela ferait disparaitre la différence entre l'heure civile usuelle, c'est-à-dire la multitude des heures locales, et l'heure officielle des chemins de fer. La loi fut votée.

La France n'avait pas eu d'heure « légale » avant la loi du 14 mars 1891 qui faisait état du seul méridien de Paris. Quelque six ans après la Conférence de Washington, cela ressemblait un peu à une provocation chauvine, mais restons positifs. "Cette loi, écrit L. De Busschère, démontre le besoin qu'en France comme ailleurs on éprouve d'unifier

13- Charles LALLEMAND, "L'heure légale et les fuseaux horaires », Revue scientifique, 16 avril 1898, p. 491-497 ; Edgar MAREUSE, «L'heure nationale », ibid., p. 690-691; Camille FLAMMARION, "La nouvelle heure de la France », Bulletin de la société astronomique de France, 1911, p. 170-175; Ambrosius Arnold Willem HUBRECHT, « Avantages économiques et hygiéniques d'un nouveau changement d'heure en France», Revue générale des sciences, 15 décembre 1912, 11 p. ; René BAILLAUD, «Le méridien de l'Observatoire de Greenwich ne sera plus le méridien origine. Le méridien origine sera pourtant toujours le méridien de Greenwich ", Annales française de chronométrie, série 2, 1, 1947, p. 161-172 ; du même, «Les États européens ont-ils pratiquement adopté l'heure définie d'après les accords internationaux par le fuseau de l'Europe centrale ?", ibid., série 2, 3, 1949, p. 255-260; Lucien BAILLAUD, « Compartimentación del tempo y cronobiología », Vigilia-Sueño, 10 (1), 1998, p. 31-37 ; du même, " La chronobiologie et l'heure civile : les domaines de la raison et des assertions hasardées ", Rythmes, à l'impression ; du même, "Heure légale et chronobiologie ", Académie des sciences, belleslettres et arts de Besançon, procès-verbaux et mémoires, sous presse, 198, p. 49-68. 
l'heure de la vie civile avec celle des chemins de fer ${ }^{14}$. » La loi officialisait comme heure légale l'heure « de la gare » qui était déjà celle de presque toute la France. Néanmoins, la circulation réelle des trains restait en retard de cinq minutes par rapport à celle-ci; ils continuaient de rouler à l'heure du méridien de Rouen. Mais on parlait de plus en plus de l'heure de Greenwich. Pour l'hydrographe Anatole Bouquet de la Grye, la préférence pour Greenwich au lieu de Paris était, en fin de compte, une courbette devant la richesse commerciale de la Grande-Bretagne. C'était une « inclinaison devant le veau d'or $»^{15}$.

Il y avait un peu plus de neuf minutes de différence entre l'heure française et celle de Greenwich ; cela entraînait la même différence avec le temps des États voisins ralliés au système des fuseaux horaires. Janssen soulignait en 1897 que «l'emploi de montres réglées sur le méridien de Paris ne cause aucune gêne aux voyageurs qui traversent notre frontière $[. .$.$] les neuf minutes de différence [. .$.$] ne font que lui$ donner une légère avance $»^{16}$. On pouvait soutenir que c'était négligeable, surtout si l'on tenait compte, dans l'autre sens, des cinq minutes de retard des horaires des trains et, aussi, de la fourchette de l'imprécision des montres de l'époque.

Les 9 et 10 décembre 1896 se tenait une conférence européenne au cours de laquelle l'administration des chemins de fer belges proposa que les chemins de fer français augmentent le retard des trains et le portent à 9 minutes; à 21 secondes près, on aurait mis les trains français non pas à l'heure de Rouen mais à celle de Greenwich ; cela ne se fit pas.

Depuis 1874, dans l'Empire allemand, aux multiples États, on établissait d'abord des horaires d'après l'heure de Berlin, puis on les traduisait, si l'on peut dire, dans les diverses heures locales. Cela n'entrainait pas d'erreurs, comme on aurait pu le craindre, mais ces complications n'étaient pas raisonnables. Le 8 octobre 1889 se réunissait le Verein für Eisenbahnkunde, qui souhaitait une heure normale pour le service intérieur et extérieur des chemins de fer, réglée sur le méridien situé à $15^{\circ}$ à l'est de celui de Greenwich. Le Verein émit le vœu que cela se généralise à l'ensemble de la vie civile de l'Empire, comme rappelait-il, en Angleterre, Suède, Amérique du Nord, et au Japon ; mais il fut ensuite décidé que la réforme n'aurait pas lieu avant

14- Louis DE BUSSCHÈRE, art. cité, 1891.

15- Anatole BOUQUET de La GRYE, «L'heure nationale », Revue scientifique, 7 mai 1898, p. $579-581$.

16- Jules JANSSEN, «La question du choix d'un méridien initial », Bulletin de la société astronomique de France, 1897, p. 146-149. 
que la vie civile se soit conformée à cette manière de compter le temps. La Prusse inaugura l'adhésion au système des fuseaux horaires, pour le service intérieur des chemins de fer, le $1^{\text {er }}$ juin $1891^{17}$.

C'est le $1^{\text {er }}$ avril 1893 que l'heure régulatrice liée au deuxième fuseau fut adoptée pour tous les usages de la vie publique et privée dans l'Allemagne entière ainsi que l'Autriche-Hongrie : heure de l'Europe centrale, ce que nous appellerions aujourd'hui l'heure UTC +1 . L'Allemagne avait une très grande étendue en longitude; la réforme des heures simplifiait l'organisation des chemins de fer; elle apportait un effet secondaire inattendu: on a constaté que la différence entre heure légale, nouvelle, et heure locale, ancienne, s'accompagnait de nettes différences dans la consommation de gaz, augmentée à l'ouest de l'Allemagne, où la nouvelle heure officielle retardait celle de la vie civile, diminuée à l'est, là où l'heure nouvelle avançait la vie civile. L'adoption de l'heure d'un fuseau pouvait passer pour de pure forme, mais elle avait des conséquences sur les dépenses d'énergie. Dès 1906, le Hollandais Hubrecht proposait que certains États choisissent leur méridien de référence en fonction des conséquences économiques de leur choix. N'insistons pas sur cette découverte, dont les chemins de fer étaient les responsables, mais dont ils n'avaient pas à se préoccuper.

Tous les pays, les uns après les autres, se mirent à l'heure de Greenwich décalée d'un nombre entier d'heures. La France allait-elle le faire elle aussi ? Comme nous avions une heure « légale » depuis 1891, il fallait une décision du législateur lui-même pour en changer. Un député, Gabriel Deville, fit en 1896 une proposition de loi pour régler l'heure sur le méridien de Greenwich. Repoussée. Une autre proposition fut faite en 1898 par le député Boudenoot, demandant que l'heure légale soit celle de Paris retardée de 9 minutes et 21 secondes; sous cette forme enveloppée, la proposition n'engendra aucune discussion à la Chambre des députés ; l'urgence fut décidée, « urgence » dérisoire : il s'écoula plus de treize années d'hésitations avant que la loi soit promulguée le 9 mars 1911. La loi annonçant le ralliement masqué à l'heure de Greenwich, retardant l'heure légale de 9 minutes et 21 secondes par rapport au méridien de Paris, s'accompagnait d'un arrêté du soussecrétaire d'État aux Postes et Télégraphes «sur la proposition du directeur de l'exploitation télégraphique »; le texte d'application aux chemins de fer de l'État figure dans le même numéro du Journal officiel du 9 mars 1911, p. 1873, mais, différence de statut, dans la rubrique

17- Giuseppe ROCCA, "Les fuseaux horaires en Europe », Bulletin de la commission internationale du congrès des chemins de fer, avril 1897, p. 649-666, p. 650. 
« informations » de la partie non administrative du J.O. ; le texte fait état de la nouvelle loi et annonce que « à partir de cette même date la différence de cinq minutes qui existe entre les horloges intérieures et extérieures des gares sera supprimée [...] les horloges extérieures seront retardées de neuf minutes et les horloges intérieures de quatre minutes ». Les chemins de fer affectent d'ignorer les durées plus courtes que la minute.

L'heure légale de la France était presque identique à celle de nos voisins ; il s'en fallait de quelques centièmes de seconde, négligeables pour la vie courante et pour les horaires des trains. Cela a duré jusqu'à la loi de 1977 qui définit l'heure légale d’après l’heure «UTC ». La loi française n'a jamais mentionné le nom détesté de Greenwich. L'honneur national est sauf, et les responsables des chemins de fer ont obtenu l'unification de la notation du temps.

\section{4 heures? ou 12 et 12 ?}

Une autre question de « temps » intéressait directement les chemins de fer. La tradition était de compter jusqu'à 12 les 12 heures avant midi ainsi que les 12 heures après midi. Les administrations françaises disaient « du matin » et « du soir »; les télégrammes, en France, portaient, après l'heure du départ, la lettre M (matin) ou S (soir), et de même les cachets postaux. Les anglophones disent ante ou post meridiem, ce qu'ils abrègent en «a.m.» ou "p.m.», sans toujours savoir quels sont les mots ainsi raccourcis. Il y a là une petite complication pour la vie courante et pour les communications. C'est le royaume de Sardaigne qui fut le premier, en 1859, a imposer, dans les télégrammes, un décompte en une série unique de 24 heures. Pour les chemins de fer, l'indication des deux sortes d'heures, d'avant et d'après midi, était matérialisée de manière variée selon les guides horaires (par exemple par des caractères différents), mais les confusions étaient possibles.

La conférence de Washington de 1884 avait recommandé que l'heure universelle soit comptée de 0 à 24. Cela concernait les gens de sciences. Ce n'était pas dans les habitudes du public. La question se posait pour les chemins de fer. Or si le citoyen continuait à toujours lire et énoncer les heures jusqu'à 12 , pouvait-on lui demander de comprendre de manière immédiate des indications de 12 à 24 ? La première objection provient, suivant Rocca, de «l'antipathie prononcée du public pour toute nouveauté qui vient froisser ses habitudes et détruire ses préjugés. C'est là qu'on doit trouver la cause des difficultés rencontrées par toutes les réformes ». On sait en effet qu'il est particulièrement difficile de s'adapter à un nouveau système d'unités, de décomptes, et pas seulement en matière monétaire. Rocca, rappelle (d'après Houzeau) qu'il fallut 
plusieurs siècles pour arriver à l'uniformité des heures diurnes et nocturnes, plus d'un demi-siècle pour la généralisation de l'usage du temps moyen préconisé par Genève en 1780. Les débats se sont déroulés sur un ton paisible, sans polémique. Est-il possible que la nouvelle notation soit comprise du public, si elle n'est pas d'abord étendue à la vie ordinaire ? Il faudrait contraindre les usagers, quand ils consultent les horaires, à faire une opération arithmétique pour se rendre compte de l'équivalence des heures. Et Rocca de dire que celui qui est capable de déchiffrer un horaire des trains donne déjà la preuve d’une intelligence supérieure à celle qui est nécessaire pour faire mentalement une soustraction «moins douze $»^{18}$.

Robertson, de l'East Indian Railway, insiste sur le fait qu'aux Indes anglaises, on a adopté la nouvelle notation sans que cela n'ait apporté aucune perturbation, ni auprès des Anglais arrivant aux Indes et $\mathrm{y}$ trouvant un système nouveau pour eux, ni pour les autochtones, voyageurs ou employés. Il est clair, dit-il, « qu'un homme qui ne serait pas capable de supputer le résultat de 2 fois 12 , ne serait pas apte au service du chemin de fer». Il ajoute que celui qui dirait que le pays au nom duquel il parlerait ne parviendrait pas à adopter les 24 heures, attribuerait à ses compatriotes une intelligence inférieure à celle des indigènes des Indes orientales. Quant à l'opportunité du système, Robertson admettait qu'il n'est pas indispensable d'inviter quelqu'un à dîner «à 20 heures » parce que pour une invitation à dîner, « 8 heures du soir » ne peut pas être confondu avec « 8 heures du matin». Cependant un ingénieur anglais lui a dit avoir manqué un train parce qu'il avait confondu 7 heures du matin avec 7 heures du soir.

Il se posait aussi la question du cadran lui-même des horloges ; nos cadrans étaient (et sont encore) divisés en 12 et non en 24. On a envisagé une irréaliste modification technique, en construisant des horloges dont la petite aiguille faisait un tour par 24 heures, mais les nombres, trop rapprochés, étaient peu lisibles. Il était plus simple d'inscrire sur le cadran deux séries de nombres, l'une allant jusqu'à 12 et l'autre de 12 à 24. Quant aux sonneries, pour éviter le comptage fastidieux des 24 coups

18- L. SCOLARI et Giuseppe ROCCA, «Exposé de la question du cadran de 24 heures (article XV de la cinquième session du congrès) », Bulletin de la commission internationale du congrès des chemins de fer, mars 1895, p. 434-472 ; L. SCOLARI et Giuseppe ROCCA (rapporteurs), "Cadran de vingt-quatre heures », ibid., mai 1897, p. 809-827 ; L. W., «L'adoption du cadran de 24 heures sur les chemins de fer de la Belgique et de l'État indépendant du Congo », ibid., janvier 1897, p. 157-158 ; J. R., « Le cadran de 24 heures et la décimalisation de l'heure et de la circonférence », ibid., septembre 1897, p. 1495-1499. 
de minuit, on a proposé de faire sonner successivement le premier chiffre puis le second ; « $2+4$ » signifierait « 24 », mais comment représenter le zéro ? Finalement, toutes les horloges sont restées au système «12», pour les cadrans comme pour les sonneries.

Une autre objection était soulevée contre le système « 24 », c'était la prononciation du nom des heures : toutes les heures post meridiem se trouvent dotées de noms plus longs que les heures ante meridiem. C'est un vrai inconvénient, partiellement compensé par la suppression de la mention matin ou soir, réalisée en France dans les indicateurs de 1913.

\section{La décimalisation du temps et les problèmes de minutes}

Adapter la mesure du temps au système décimal est une idée qui apparait de temps en temps. La Convention nationale avait décidé en 1793 de diviser le jour en 10 heures (au lieu de 24), divisées en 100 minutes de 100 secondes. Le système a fait long feu. Actuellement, seuls les chronobiologistes, peut-être, notent les fractions de jour en chiffres décimaux après la virgule. C'est pourtant de manière tout à fait banale qu'on divise la seconde de temps en dixièmes, centièmes, etc., de seconde.

Une autre forme de décimalisation a été proposée en septembre 1897, qui pouvait concerner les chemins de fer. Une commission a été constituée à l'initiative du ministère français de l'Instruction publique, pour étudier la décimalisation du temps et de la circonférence. On a envisagé de faire commencer le système à partir de notre heure duodécimale actuelle, qui serait divisée en 100 minutes de 100 secondes $^{19}$. D’après Giuseppe Rocca cela aurait un certain intérêt pour les chemins de fer, en facilitant les calculs de durée des parcours, de vitesse des trains, etc. Un autre avantage serait de faire des « minutes » plus courtes, qui dureraient seulement 36 de nos secondes habituelles, sexagésimales : les horaires des trains étant établis en nombres entiers de minutes, cela donnerait plus de souplesse au choix de la durée des arrêts. Mais il faudrait refaire les mécanismes des horloges et des montres, et d'autre part les cadrans seraient d'une lecture plus difficile. Rocca concluait que les chemins de fer ne s'opposeraient pas à cette réforme, mais qu'ils l'accepteraient sans aucun enthousiasme ${ }^{20}$.

19- C. J. VAN MIERLO, «L'heure décimale, comparaison des divers systèmes proposés ", Bulletin de la commission internationale du congrès des chemins de fer, février 1899, p. 171-182.

20- Giuseppe ROCCA, "L'heure décimale, le méridien initial et les fuseaux horaires », Bulletin de la commission internationale du congrès des chemins de fer, décembre 1899, p. 14831497. 
Une unité de temps se rapproche parfois de la minute ; c'est «l'instant », dont la durée, brève, dépend du contexte. Dans les annonces orales des trains, lorsque le prochain arrêt est prévu pour « dans quelques instants ", il s'agit de deux ou trois minutes ; si nous supposons qu'ici « quelques » signifie « quatre ou cinq », on peut estimer que «l'instant » de la SNCF dure à peu près une bonne demi-minute...

\section{Conclusion}

La mesure du temps est fondamentalement l'affaire des astronomes ; la vie en société a conduit à officialiser la compartimentation $\mathrm{du}$ temps, telle que nous la connaissons. Mais ce sont les communications rapides qui ont conduit à normaliser, à unifier, notre repérage dans le temps, et finalement à faire intervenir la loi dans la régulation temporelle de la société.

L'heure UTC, les horloges à affichage numérique, etc., représentent des aspects du progrès scientifique et technique, mais cela ne doit pas faire oublier que les fuseaux horaires, notre heure légale, le décompte des 24 heures, nous les devons essentiellement aux chemins de fer. 